\title{
Minor Salivary Gland Adenocarcinoma
}

National Cancer Institute

\section{Source}

National Cancer Institute. Minor Salivary Gland Adenocarcinoma. NCI Thesaurus. Code C5948.

An adenocarcinoma that arises from the minor salivary glands. 